\title{
Biocompatible polymers coated on carboxylated nanotubes functionalized with betulinic acid for effective drug delivery
}

\begin{abstract}
Chemically functionalized carbon nanotubes are highly suitable and promising materials for potential biomedical applications like drug delivery due to their distinct physico-chemical characteristics and unique architecture. However, they are often associated with problems like insoluble in physiological environment and cytotoxicity issue due to impurities and catalyst residues contained in the nanotubes. On the other hand, surface coating agents play an essential role in preventing the nanoparticles from excessive agglomeration as well as providing good water dispersibility by replacing the hydrophobic surfaces of nanoparticles with hydrophilic moieties. Therefore, we have prepared four types of biopolymer-coated single walled carbon nanotubes systems functionalized with anticancer drug, betulinic acid in the presence of Tween 20, Tween 80 , polyethylene glycol and chitosan as a comparative study. The Fourier transform infrared spectroscopy studies confirm the bonding of the coating molecules with the SWBA and these results were further supported by Raman spectroscopy. All chemically coated samples were found to release the drug in a slow, sustained and prolonged fashion compared to the uncoated ones, with the best fit to pseudosecond order kinetic model. The cytotoxic effects of the synthesized samples were evaluated in mouse embryonic fibroblast cells (3T3) at 24, 48 and $72 \mathrm{~h}$. The in vitro results reveal that the cytotoxicity of the samples were dependent upon the drug release profiles as well as the chemical components of the surface coating agents. In general, the initial burst, drug release pattern and cytotoxicity could be well-controlled by carefully selecting the desired materials to suit different therapeutic applications.
\end{abstract}

Keyword: Drug delivery; Coatings; Carboxylated nanotubes 